\title{
EDITORIAL
}

\section{Update on the European Respiratory Review}

\author{
M. Humbert
}

$\mathbf{T}$ his editorial serves as a progress report on the European Respiratory Review, 2 years into my term as chief editor, and with the Review now being established in its current format, which was introduced to readers in March 2009 [1].

Over this period, the European Respiratory Review has proven a success, with the new European Respiratory Update feature being particularly popular among readers. The purpose of these articles is to summarise the recent developments in a particular field of respiratory health and disease made in the previous 1 or $2 \mathrm{yrs}$; they also serve as a focal point for each issue of the Review, often being accompanied by complementary reviews on related subject matter. In the first of these updates, published in March 2009, SOUZA and JARDIM [2] described the results of clinical trials and epidemiological studies in pulmonary arterial hypertension, and called for international collaborations to help standardise the reporting and study of this rare disease, in order to direct future studies and help to delineate their findings. This was complemented by a review on the subject of high-altitude pulmonary hypertension [3] and a series of short reviews of pulmonary hypertension therapies [4-7]. Following this, GAGA et al. [8] provided an update on the epidemiology, clinical assessment, risk factors, pathophysiology and management of severe asthma in June 2009. Again, it was recognised that a coordinated global effort was needed, this time to help asthma patients with the most severe form of the disease. Elsewhere in that issue of the Review, the theme was developed by a summary of expert workshop presentations on the role of distal airways in asthma [9] and a report from studies of asthma control across Europe [10]. September 2009 saw SANCHEZ et al. [11] provide their commentary on past and potential future developments in venous thromboembolic disease, with a focus on risk factors, diagnosis, prognosis and treatment of acute pulmonary embolism. The next subject to feature in the update series was chronic obstructive pulmonary disease (COPD). ROCHE and co-workers [12, 13] addressed COPD, and the vast body of constantly updating literature pertaining to it, over the course of two consecutive updates published in December 2009 and March 2010. The first part examined the more fundamental aspects of pathophysiology, lung mechanics and gas exchange,

\footnotetext{
*Université Paris-Sud, Faculté de Médecine, Kremlin-Bicêtre, AP-HP, Centre National de Référence de l'Hypertension Pulmonaire Sévère, Service de Pneumologie et Réanimation Respiratoire, Hôpital Antoine Béclère, Clamart, and INSERM U999, Hypertension Artérielle Pulmonaire, Physiopathologie et Innovation Thérapeutique, IPSIT, Centre Chirurgical Marie-Lannelongue, Le Plessis-Robinson, France.

CORRESPONDENCE: M. Humbert, Service de Pneumologie et Réanimation Respiratoire, Hôpital Antoine Béclère, Assistance-Publique-Hôpitaux de Paris, Université Paris-Sud 11, 157 rue de la Porte de Trivaux, 92140 Clamart, France. E-mail: marc.humbert@abc.aphp.fr
}

and placed these in the context of clinical topics, such as the respiratory symptoms of COPD and its comorbidities. In the same issue, the epidemiological aspects of the disease were reviewed in a manuscript authored by RAHERISON and GIRODET [14]. The second part of the update went on to review COPD therapies, from pharmacology- to instrument-based treatments. Alongside this presentation of the advances made in COPD therapies, the difficulty in treating patients and designing trials for such a heterogeneous disease was discussed, and the need for not only the publication of treatment guidelines, but a more universal adherence to them, was emphasised. Also featured in the March 2010 issue was a systematic review of the effects of Heliox therapy on exercise performance and dyspnoea perception in COPD patients [15], as well as a discussion of the mechanisms by which pulmonary rehabilitation can benefit those with COPD, and how this benefit may extend to a wider population in the future [16]. Important advances in the understanding of the diffuse parenchymal lung diseases were communicated by HARARI and CAMiNATi [17] in June 2010, with particular interest in the progress made in the field of idiopathic pulmonary fibrosis. The most recent update to be published in the European Respiratory Review, written by ZALCMAN et al. [18], covers nonsmall cell lung cancer, providing a summary of recent results from chemotherapy and chemoradiotherapy trials. New treatment regimens and latest developments in disease sub-categorisation, and the resultant targeted therapies, are comprehensively detailed, along with the ground-breaking increases in overall patient survival that they have enabled. The update also reports on the latest research presented at the most recent meeting of the American Society of Clinical Oncology, in Chicago, IL, USA in June 2010, ensuring that the material included is as up to date as possible. The lung cancer theme was extended to a review of gefitinib therapy across a number of trials in nonsmall cell lung cancer, which highlighted the challenges involved in drug development and targeting chemotherapy to very specific subsets of patients, in order to provide the most effective and safe treatment [19].

Looking to the future, we will next feature another update in the fast-growing field of pulmonary hypertension, in which R. Souza will review research and clinical findings published in 2009 and 2010. We have also already scheduled the European Respiratory Update articles for 2011. Look out for updates on tuberculosis (O.M. Kon), sleep-related disorders (P. Levy), granulomatous lung diseases (G. Raghu) and pulmonary infections (T. Welte) during the year.

As well as refreshing the Review with its current format, one of my other goals as chief editor was to develop and consolidate its relationship with the flagship publication of the European 
Respiratory Society (ERS), the European Respiratory Journal. To this end, an online submission platform (http://mc. manuscriptcentral.com/err) was set up at the beginning of 2009, and now allows users to move easily between publications and even transfer their submitted manuscript to another of the ERS journals if they so wish. In addition, the ERS has relaunched online access to its journals, with brand new Highwire-hosted sites at www.ersjournals.com providing a new user-friendly interface and greater functionality; the European Respiratory Journal, Review and Monograph benefit from a higher degree of integration, search facilities available across all three publications and the new ERS subject collections, which group ERS articles through common themes rather than by journal or publication date. A further appropriate example of the partnership between the European Respiratory Journal and the Review is the contemporaneous publication of the European Society of Cardiology/ ERS guidelines statement for the management of pulmonary hypertension, in the European Respiratory Journal [20], and a complementary article in the European Respiratory Review on the interpretation of these guidelines in a real-life clinical setting [21].

I am pleased to report that these changes to the European Respiratory Review have been positively received in the community and we now are happily seeing a steady flow of unsolicited manuscripts being submitted through our online platform and an increase in the number of visits to our website. We are particularly pleased to confirm that, after a period of evaluation, the National Library of Medicine (at the National Institutes of Health, Bethesda, MD, USA) has selected the European Respiratory Review for inclusion in its MEDLINE database. The Review can now take its place, alongside the European Respiratory Journal and over 5,000 of the most highly regarded medical science publications, in the most widely used bibliographic database in the field. We are delighted with this achievement, as the exposure of the Review will be greatly increased by its inclusion in MEDLINE and subsequent visibility through the PubMed search platform. Such an achievement has been possible thanks to the fantastic involvement of the members of the editorial board as well as the friendly support of the European Respiratory Journal chief editors, A.T. Dinh-Xuan and V. Brusasco. Last but not least, the ERS publications office has been always supportive and flexible, allowing timely publication of high-quality material. I wish to warmly thank the whole team for such a professional contribution to these recent achievements.

\section{STATEMENT OF INTEREST}

A statement of interest for M. Humbert can be found at www.erj. ersjournals.com/site/misc/statements.xhtml

\section{REFERENCES}

1 Humbert M. Introducing the new European Respiratory Review. Eur Respir J 2009; 33: 466-467.

2 Souza R, Jardim C. Trends in pulmonary arterial hypertension. Eur Respir Rev 2009; 18: 7-12.

$3 \mathrm{Xu} \mathrm{W-Q,} \mathrm{Jing} \mathrm{Z-C.} \mathrm{High-altitude} \mathrm{pulmonary} \mathrm{hypertension.} \mathrm{Eur}$ Respir Rev 2009; 18: 13-17.

4 Beghetti M. Paediatric pulmonary hypertension: monitoring progress and identifying unmet needs. Eur Respir Rev 2009; 18: 18-23.

5 Lang IM. Managing chronic thromboembolic pulmonary hypertension: pharmacological treatment options. Eur Respir Rev 2009; 18: $24-28$

6 Olschewski $\mathrm{H}$. Inhaled iloprost for the treatment of pulmonary hypertension. Eur Respir Rev 2009; 18: 29-34.

7 Ghofrani HA, Grimminger F. Soluble guanylate cyclase stimulation: an emerging option in pulmonary hypertension therapy. Eur Respir Rev 2009; 18: 35-41.

8 Gaga M, Zervas E, Chanez P. Update on severe asthma: what we know and what we need. Eur Respir Rev 2009; 18: 58-65.

9 Burgel P-R, de Blic J, Chanez P, et al. Update on the roles of distal airways in asthma. Eur Respir Rev 2009; 18: 80-95.

10 Demoly P, Paggiaro P, Plaza V, et al. Prevalence of asthma control among adults in France, Germany, Italy, Spain and the UK. Eur Respir Rev 2009; 18: 105-112.

11 Sanchez O, Planquette B, Meyer G. Update on acute pulmonary embolism. Eur Respir Rev 2009; 18: 137-147.

12 Bourdin A, Burgel P-R, Chanez P, et al. Recent advances in COPD: pathophysiology, respiratory physiology and clinical aspects, including comorbidities. Eur Respir Rev 2009; 18: 198-212.

13 Alifano M, Cuvelier A, Delage A, et al. Treatment of COPD: from pharmacological to instrumental therapies. Eur Respir Rev 2010; 19 7-23.

14 Raherison C, Girodet P-O. Epidemiology of COPD. Eur Respir Rev 2009; 18: 213-221.

15 Hunt T, Williams MT, Frith P, et al. Heliox, dyspnoea and exercise in COPD. Eur Respir Rev 2010; 19: 30-38.

16 Troosters T, Gosselink R, Janssens W, et al. Exercise training and pulmonary rehabilitation: new insights and remaining challenges. Eur Respir Rev 2010; 19: 24-29.

17 Harari S, Caminati A. Update on diffuse parenchymal lung disease. Eur Respir Rev 2010; 19: 97-108.

18 Zalcman G, Bergot E, Lechapt E. Update on nonsmall cell lung cancer. Eur Respir Rev 2010; 19: 173-185.

19 Armour AA, Watkins CL. The challenge of targeting EGFR experience with gefitinib in nonsmall cell lung cancer. Eur Respir Rev 2010; 19: 186-196.

20 Galiè N, Hoeper MM, Humbert M, et al. Guidelines for the diagnosis and treatment of pulmonary hypertension. Eur Respir $J$ 2009; 34: 1219-1263.

21 Montani D, O'Callaghan DS, Jaïs X, et al. Implementing the ESC/ ERS pulmonary hypertension guidelines: real-life cases from a national referral centre. Eur Respir Rev 2009; 18: 272-290. 